\title{
Magnetic states of Ni-Mn-Sn based shape memory alloy: a combined muon spin relaxation and neutron diffraction study
}

\author{
J. Sannigrahi, S. Pramanick, S. Chatterjee, J. S. Lord, \\ D. Khalyavin, A. D. Hillier, D. T. Adroja and S. Majumdar
}

\section{Published version information}

Citation: J Sannigrahi et al. "Magnetic states of Ni-Mn-Sn based shape memory alloy: a combined muon spin relaxation and neutron diffraction study." Physical Review B, vol. 99, no. 22 (2019): 224401.

DOI: $10.1103 /$ PhysRevB.99.224401

This version is made available in accordance with publisher policies. Please cite only the published version using the reference above. This is the citation assigned by the publisher at the time of issuing the APV. Please check the publisher's website for any updates. 


\title{
Magnetic states of Ni-Mn-Sn based shape memory alloy: A combined muon spin relaxation and neutron diffraction study
}

\author{
J. Sannigrahi, ${ }^{1}$ S. Pramanick, ${ }^{2}$ S. Chatterjee,${ }^{3}$ J. S. Lord,${ }^{1}$ D. Khalyavin, ${ }^{1}$ A. D. Hillier, ${ }^{1}$ D. T. Adroja,${ }^{1,4}$ and S. Majumdar ${ }^{3, *}$ \\ ${ }^{1}$ ISIS Neutron and Muon Source, Science and Technology Facilities Council, Rutherford Appleton Laboratory, \\ Didcot OX11 0QX, United Kingdom \\ ${ }^{2} U G C$-DAE Consortium for Scientific Research, Kolkata Centre, Sector III, LB-8, \\ Salt Lake, Kolkata 700 098, India \\ ${ }^{3}$ School of Physical Sciences, Indian Association for the Cultivation of Science, $2 A$ \& B Raja S. C. Mullick Road, \\ Jadavpur, Kolkata 700 032, India \\ ${ }^{4}$ Highly Correlated Matter Research Group, Physics Department, University of Johannesburg, Auckland Park 2006, South Africa
}

(Received 15 February 2019; revised manuscript received 1 May 2019; published 3 June 2019)

\begin{abstract}
The fascinating multiple magnetic states observed in the Ni-Mn-Sn based metamagnetic shape memory alloy are addressed through a combined muon spin relaxation $(\mu \mathrm{SR})$ and neutron powder diffraction study. The material used in the present investigation is an off-stoichiometric alloy of nominal composition, $\mathrm{Ni}_{2.04} \mathrm{Mn}_{1.4} \mathrm{Sn}_{0.56}$. This prototypical alloy, similar to other members in the Ni-Mn-Sn series, orders ferromagnetically below $T_{C A}$ $(=320 \mathrm{~K})$ and undergoes a martensitic-type structural transition at $T_{M S}(=290 \mathrm{~K})$, which is associated with the sudden loss of magnetization. The sample regains its magnetization below another magnetic transition at $T_{C M}=260 \mathrm{~K}$. Eventually, the composition shows a steplike anomaly at $T_{B}=120 \mathrm{~K}$, which is found to coincide with the blocking temperature of the exchange bias effect observed in the alloy. In our study, the initial asymmetry $A_{10}$ of the $\mu \mathrm{SR}$ data falls rapidly below $T_{C A}$, indicating the onset of bulk magnetic order. $A_{10}$ regains its full asymmetry value below $T_{M S}$, suggesting the collapse of the ferromagnetic order into a fully disordered paramagnetic state. Below the second magnetic transition at $T_{C M}$, asymmetry drops again, confirming the reentrance of a long-range ordered state. Interestingly, $A_{10}$ increases sluggishly below $T_{B}$, indicating that the system attains a disordered/glassy magnetic phase below $T_{B}$, which is responsible for the exchange bias and frequency dispersion in the ac susceptibility data as previously reported. The neutron powder diffraction data do not show any magnetic superlattice reflections, ruling out the possibility of a long-range antiferromagnetic state at low temperatures. The ground state is likely to be comprised of a concentrated metallic spin glass in the backdrop of an ordered ferromagnetic state.
\end{abstract}

DOI: 10.1103/PhysRevB.99.224401

\section{INTRODUCTION}

Advanced functional materials play an ever-increasing role in modern technological developments, which encompass areas such as energy harvesting, computation, and communication, as well as combating environmental pollution. Broadly, they can be classified into five groups depending upon their functionality: adaptive, magnetic, electric, optical, and energy and environmental materials. It is needless to mention that a proper investigation of their physical properties is important in understanding the essential physics associated with their functionality. For example, the study of CuO-based high$T_{C}$ superconductors has enriched our understanding of the electronic properties of correlated metal oxides. Many of these functional materials are actually multifunctional; that is, they show two or more functional properties. $\mathrm{Ni}_{2.04} \mathrm{Mn}_{1.4} \mathrm{Sn}_{0.56}$, the title composition of this work, is one such material having functionality as an adaptive, magnetic, electric, and energy and environmental material.

\footnotetext{
*sspsm2@iacs.res.in
}

$\mathrm{Ni}_{2.04} \mathrm{Mn}_{1.4} \mathrm{Sn}_{0.56}$ belongs to a class of materials known as metamagnetic shape memory alloys (MSMAs). They show large magnetic-field-induced strain, magnetoresistance, magnetocaloric, barocaloric, and exchange bias effects [1-6]. The general compositions of these alloys can be expressed as $\mathrm{Ni}_{2} \mathrm{Mn}_{1+p} Z_{1-p}(Z=\mathrm{In}, \mathrm{Sn}, \mathrm{Sb}$ and $p<1)$, and the observed functionality arises from their biferroic nature with the simultaneous presence (as well as the mutual interplay) of ferromagnetism and ferroelasticity. The alloys are characterized by a first-order martensitic-type structural phase transition occurring at a temperature $T_{M S}$ and a second-order paramagnetic (PM) to ferromagnetic (FM) transition at the Curie point $T_{C}$. For the practical realization of magnetic shape memory and other magnetofunctional properties, one should have $T_{C}>T_{M S}$; that is, the sample should be in a magnetically ordered state when martensitic phase transition (MPT) takes place.

The presence of two critical temperatures (namely, $T_{C}$ and $T_{M S}$ ) makes the system have a rather exotic phase diagram. There are a few important aspects associated with these alloys, which remain elusive to date. First, what happens to the FM state of the alloy (we are considering $T_{C}>T_{M S}$ ) right 
below the MPT? The high-temperature structural phase is called austenite with cubic lattice symmetry, while the lowtemperature phase (below $T_{M S}$ ) is called martensite, with a tetragonal/orthorhombic/modulated structure. It is found that magnetization drops sharply below $T_{M S}$, indicating the loss of the ordered FM moment. Some diffuse peaks were observed in the neutron powder diffraction (NPD) data of Ni-Mn-Sn alloys, which were attributed to the existence of incipient antiferromagnetic (AFM) coupling [7]. As is evident from the chemical compositions, a fraction of $Z$ atoms is replaced by $\mathrm{Mn}$ (we call it $\mathrm{Mn}^{\prime}$ ), and theoretical calculations indicate the existence of AFM correlation between regular Mn and $\mathrm{Mn}^{\prime}$ atoms [8-14]. On the other hand, ${ }^{57} \mathrm{Co}-\mathrm{Rh}$ Mössbauser spectroscopy on a Ni-Mn-Sn alloy (with a small amount of enriched ${ }^{57} \mathrm{Fe}$ doped at the $\mathrm{Mn}$ site) indicated a PM state below $T_{M S}$ of the sample [15]. NPD studies, performed on various $\mathrm{Ni}-\mathrm{Mn}-Z$ alloys, fail to identify well-defined magnetic Bragg peaks associated with an ordered AFM state $[7,16]$. Neutron polarization study indicates the existence of FM correlations, which vanish below $T_{M S}$ with the concomitant occurrence of $\mathrm{Mn}-\mathrm{Mn}^{\prime}$ AFM correlations [17]. Therefore, the nature of the magnetic state just below $T_{M S}$ remains uncertain; it may be incipient AFM in the backdrop of a PM/FM phase, a long-range ordered AFM state with a weak moment, or an FM state with a reduced $\mathrm{Mn}$ moment.

The second unresolved point is associated with the magnetic ground state of these alloys. The off-stoichiometric Ni$M n-Z$ alloys show a steplike feature well below $T_{M S}$ in the zero-field-cooled magnetization data, and an exchange bias (EB) effect was observed below $T_{B}$. Our group previously reported that field cooling from just above $T_{B}$ is sufficient to observe EB, and $T_{B}$ actually signifies a spin-freezing temperature [18]. Subsequently, there have been numerous reports on the glassy magnetic state of the Ni-Mn- $Z$ alloys [19-22]. Nevertheless, ambiguities remain about the nature of the glassy state, and the ground state has been described as a reentrant spin glass, cluster glass, and superspin glass by various authors (cited in the previous sentence). EB generally requires two different magnetic phases (say, FM + AFM or spin glass + FM) to be present. The presence of AFM clusters below $T_{M S}$ is highly possible since there is strong evidence for incipient antiferromagnetism. It remains unclear whether the ground state is characterized by (i) a mixture of AFM and FM phase fractions along with interfacial glassiness, (ii) coexisting spin-glass (SG) and FM phases, or (iii) a standalone SG phase.

In the present work, we address these aspects using muon spin resonance/rotation $(\mu \mathrm{SR})$ as well as NPD techniques on an MSMA of nominal composition, $\mathrm{Ni}_{2.04} \mathrm{Mn}_{1.4} \mathrm{Sn}_{0.56}$. It is to be noted that Brown et al. [7] studied a similar sample $\left(\mathrm{Ni}_{2} \mathrm{Mn}_{1.44} \mathrm{Sn}_{0.56}\right)$ using NPD. In comparison to Brown et al.'s sample, $2 \%$ of excess $\mathrm{Ni}$ is doped at the expense of $\mathrm{Sn}$, which elevates $T_{M S}$ by about $70 \mathrm{~K}$. While the ferromagnetic Curie point of the presently studied sample high-temperature austenite is around $T_{C A}=320 \mathrm{~K}$, the structural transition takes place around $T_{M S}=290 \mathrm{~K}$. The reason for choosing this composition lies in the fact that $T_{M S}$ is very close to $T_{C A}$, and the FM state becomes unstable below the MPT (see Fig. 1). The sample regains its magnetization below a second transition at $T_{C M}=260 \mathrm{~K}$ in the martensitic phase. The steplike anomaly
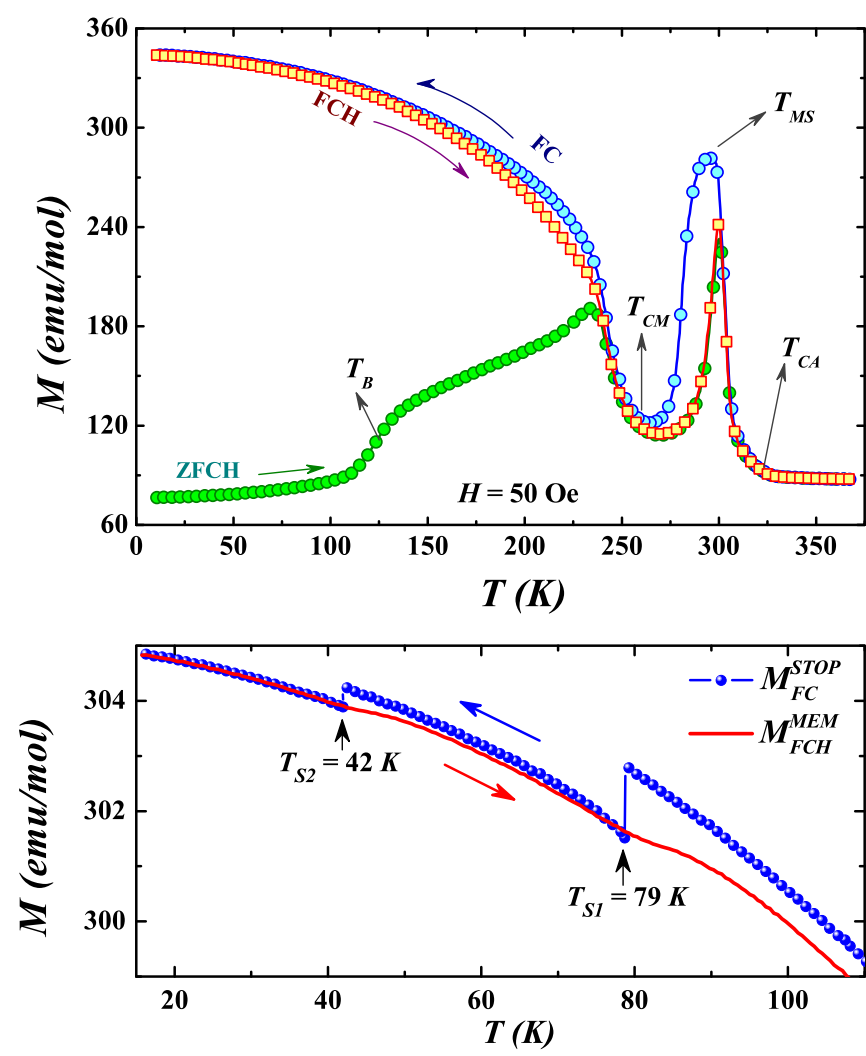

FIG. 1. In the top panel, magnetization is plotted as a function of temperature in zero-field-cooled heating ( $\mathrm{ZFCH})$, field cooling (FC), and field-cooled heating (FCH) conditions in the presence of 50 Oe of external magnetic field. The martensitic transformation $T_{M S}$ is clearly visible around $300 \mathrm{~K}$, where large thermal hysteresis is present. $T_{C A}, T_{C M}$, and $T_{B}$ indicate the austenite Curie point, martensite Curie point, and exchange bias blocking temperature, respectively. The bottom panel shows the field-cooled-field-stop memory data, indicating the presence of a glassy magnetic phase below $120 \mathrm{~K}$. Here $M_{\mathrm{FC}}^{\mathrm{STOP}}$ denotes the magnetization data recorded during cooling along with 1 -h stops at 80 and $40 \mathrm{~K}$. Magnetic field was turned off during the stops. $M_{\mathrm{FCH}}^{M E M}$ denotes the subsequent heating run, and clear anomalies (in the form of shallow dips) were observed at the stopping points, signifying the presence of memory.

is seen below $T_{B}=120 \mathrm{~K}$, and considerable EB is observed at low temperature [23]. The sample shows the signature of field-cooled-field-stop memory (FCFS) [24], as shown in the bottom panel of Fig. 1. The observed FCFS in this bulk sample indicates the presence of a glassy magnetic ground state. The reentrant spin-glass state comes from magnetic frustration due to the presence of short-range AFM correlations coexisting with the FM state, as observed in a similar Ni-Mn-Sn sample by Aksoy et al. [17].

\section{EXPERIMENTAL DETAILS}

The polycrystalline sample of $\mathrm{Ni}_{2.04} \mathrm{Mn}_{1.4} \mathrm{Sn}_{0.56}$ for the present study was prepared by argon arc melting the constituent elements [23]. The temperature $T$-dependent dc magnetization $M$ measurements were performed using a commercial Quantum Design superconducting quantum interference device magnetometer (MPMS 3). The $\mu \mathrm{SR}$ measurements 
were performed at the ISIS Facility, Rutherford Appleton Laboratory, United Kingdom, using EMU (for zero magnetic field) and HIFI (for longitudinal magnetic field) spectrometers. The sample was mounted on a silver sample holder to minimize the background, and measurements were performed at different temperatures. The neutron powder diffraction was carried out at the WISH time-of-flight diffractometer at the ISIS facility between 8 and $363 \mathrm{~K}$. The powdered sample was inserted in a cylindrical vanadium container $6 \mathrm{~mm}$ in diameter. A standard helium closed-cycle refrigerator was used to cool the sample down to $8 \mathrm{~K}$. Nuclear and magnetic structure refinements were performed by the Rietveld method using the FULLPROF program [25]. The diffraction peaks were described by a pseudo-Voigt profile function. All the measurements mentioned above were performed on the sample obtained from a single batch.

\section{RESULTS}

\section{A. Muon spin relaxation}

$\mu \mathrm{SR}$ is an accomplished local probe technique to study the magnetism of a material [26]. Spin-polarized positive muons $\mu^{+}$are implanted into the sample. The implanted muons decay into positrons, which are emitted preferentially in the direction of the muon spin. The spin of the implanted $\mu^{+}$ precesses around the effective magnetic field vector at the site of implantation. Random and fluctuating fields within the sample can depolarize the muons. In the actual zero-field (ZF) or longitudinal field (LF) experiment, the emitted positrons are counted parallel and antiparallel to the initial muon spin direction. The difference between the number of positrons in the forward and backward directions is generally measured as a function of time $t$, and it is called the asymmetry function $G_{z}(t)$. Since $G_{z}(t)$ is a measure of muon depolarization, one can get significant information on the magnetic state of the material out of it $[27,28]$.

For a typical magnetic material, the relaxing part of the asymmetry often obeys an exponential law, $G_{z}=$ $A_{0} \exp (-\lambda t)$, where $A_{0}$ is the initial asymmetry and $\lambda$ is the (spin-lattice) relaxation rate. For the present Ni-Mn-Sn alloy, the simple exponential law is found to be inadequate to describe the data at all temperatures. The simplest approach is to have a bimodal distribution with two relaxation rates, $\lambda_{1}$ and $\lambda_{2}$, which results in [29]

$$
G_{z}(t)=A_{10} \exp \left(-\lambda_{1} t\right)+A_{20} \exp \left(-\lambda_{2} t\right)+b_{g} .
$$

Here $b_{g}$ denotes the time-independent background of asymmetry. A similar two-exponent model has been used for diverse magnetic systems successfully, which include perovskite manganites [29-31], cobaltates [32], and Heuslerbased intermetallic alloys [33]. The regression converges better over the full $T$ range if we set the constraint $A_{20}=$ $0.4 A_{10}$, and the fittings presented in the subsequent parts were performed considering the above constraint. The value of $b_{g}$ for a particular fixed magnetic field was estimated from $T=30 \mathrm{~K}$ data, and it is kept constant at all $T$ for a particular value of $H$. For the $\mathrm{ZF}$ case, the value of $b_{g}$ was kept constant at 0.0094(4), while for the LF data it was fixed at the value $0.1973(4)$.
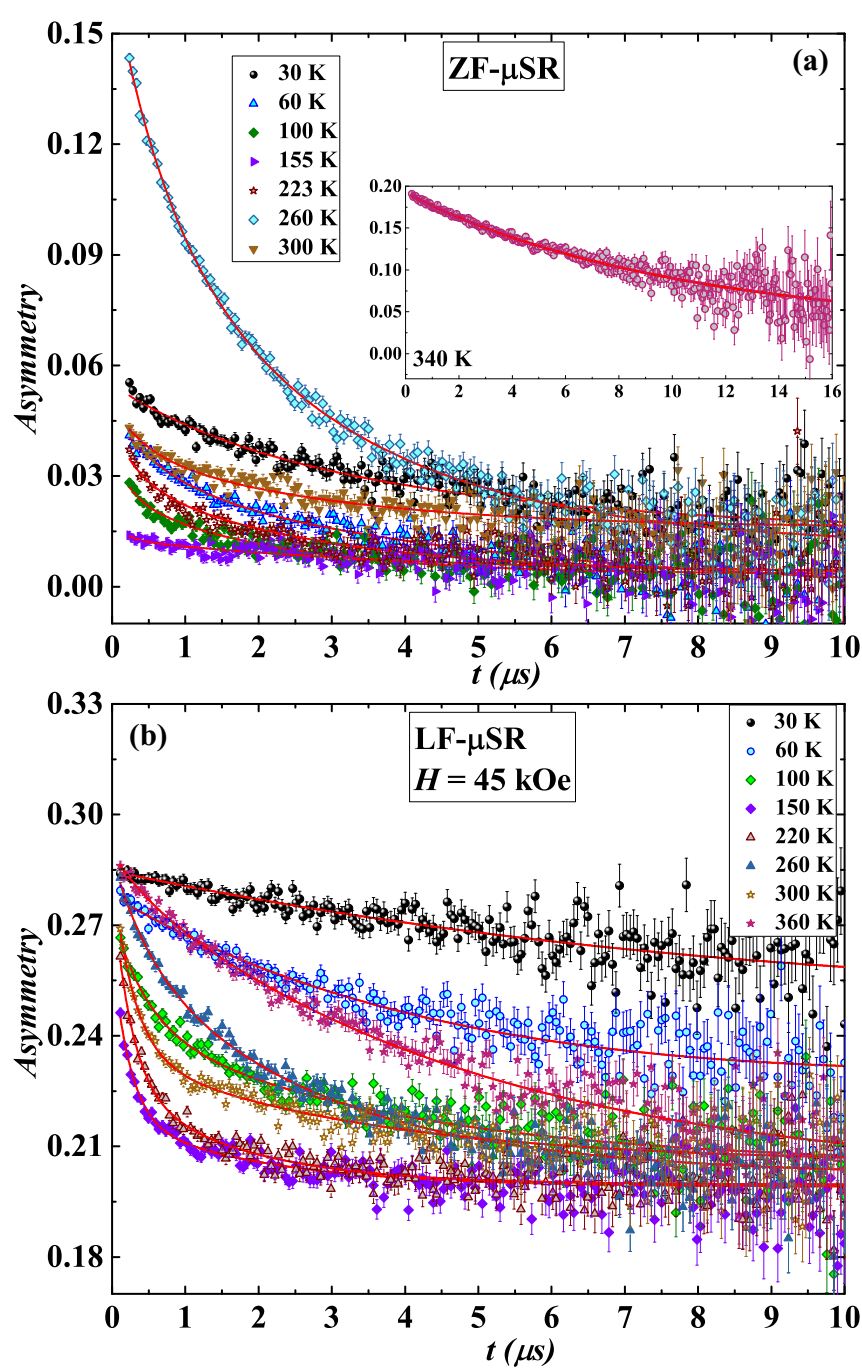

FIG. 2. (a) The muon spin relaxation data measured in zero magnetic field at different constant temperatures. The inset shows the same recorded at $340 \mathrm{~K}$, where the sample is in the paramagnetic austenitic phase. (b) Similar data recorded in the presence of $45 \mathrm{kOe}$ of applied magnetic field.

Considering the glassy ground state in the studied alloy, we have additionally used a stretched exponential function to fit the data below $150 \mathrm{~K}$ [27,34-37],

$$
G_{z}(t)=A_{10} \exp \left[(-\lambda t)^{\beta}\right]+b_{g} .
$$

Here $\beta$ is called the shape parameter. Like for the doubleexponential fitting, we have kept $b_{g}$ fixed for all $T$ values for a particular $H$.

Figure 2(a) shows the time domain $\mu \mathrm{SR}$ data recorded at different temperatures in the ZF condition while cooling. At $T=340 \mathrm{~K}$, the asymmetry shows an exponential-like decay, which is expected for a PM state. On lowering the temperature below the FM Curie point $\left(T_{C A}=320 \mathrm{~K}\right), G_{z}(t)$ shows a fast relaxation component, which coexists with the slow relaxing part. This is clearly due to the onset of FM transition in the system. On further lowering $T$ below $T_{M S}(=290 \mathrm{~K})$, the fast relaxation component appears to get diminished. This corresponds to the rapid fall of $M$ below $T_{M S}$ in the magnetization 


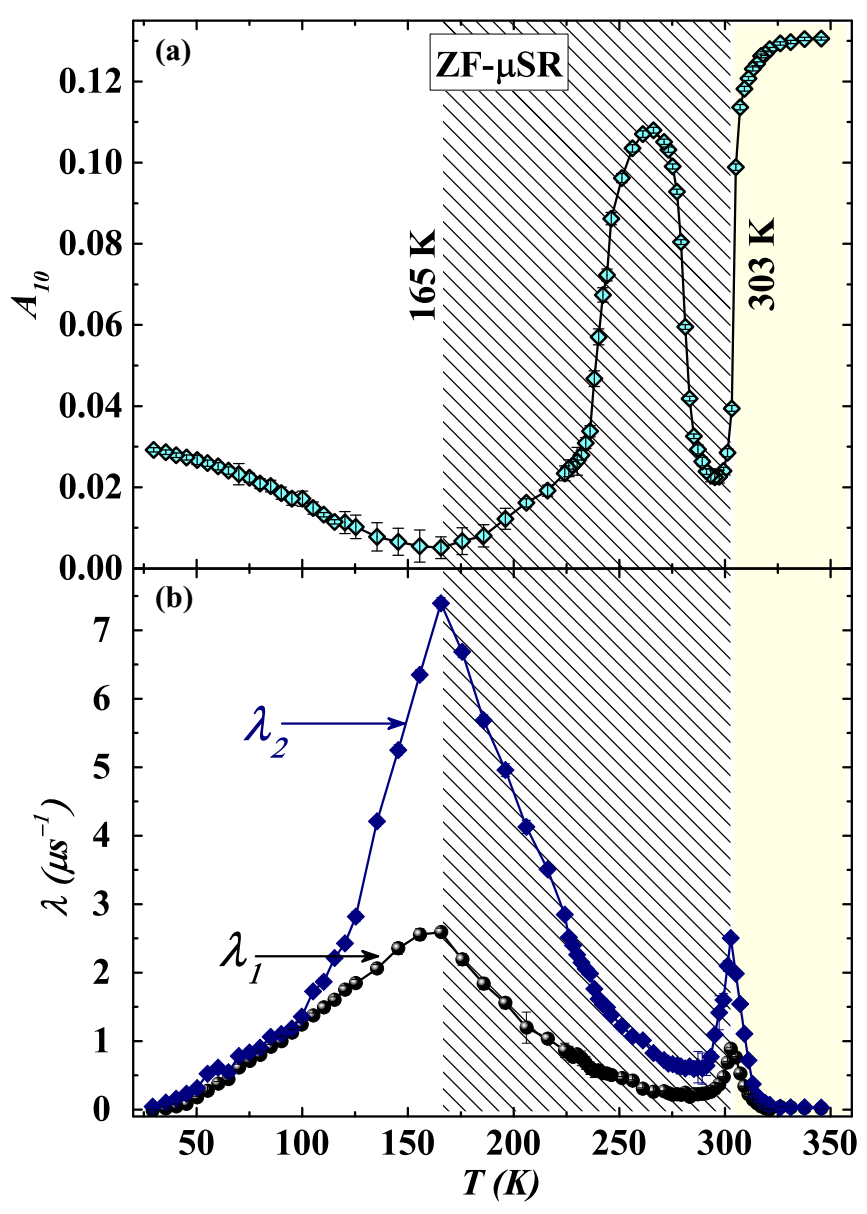

FIG. 3. (a) The muon asymmetry $A_{10}$ as a function of temperature measured in zero magnetic field while cooling. (b) The thermal variation of the muon depolarization rate $\lambda_{1}$ and $\lambda_{2}$ at $H=0$.

data (see Fig. 1, top panel). The fast relaxation reappears when the sample is cooled below the second magnetic ordering point $T_{C M}$. Most interestingly, the damping gradually gets reduced when the sample is cooled below the blocking temperature, $T_{B}=120 \mathrm{~K}$. We also recorded the $\mu \mathrm{SR}$ spectra in the presence of $45 \mathrm{kOe}$ of applied longitudinal field, as depicted in Fig. 2(b).

In order to elucidate the magnetic state of the sample at different $T$, we have fitted the time domain data with the double-exponential function stated in Eq. (1). The solid lines in Figs. 2(a) and 2(b) represent the fit to the data. The values of $A_{10}$ and $\left(\lambda_{1}, \lambda_{2}\right)$, obtained by fitting the ZF data, are plotted in Figs. 3(a) and 3(b), respectively.

The $T$ variation of $A_{10}$ [see Fig. 3(a)] provides noteworthy information on the magnetic state of the studied alloy. In the PM state (above $310 \mathrm{~K}$ ), the initial asymmetry is found to be almost $T$ independent. On cooling, $A_{10}$ falls sharply with the onset of ferromagnetism at $T_{C A}$, and it attains its lowest value just below $300 \mathrm{~K}$. The structural transition is beautifully echoed in that data, as $A_{10}$ again rises sharply below $T_{M S}$ and attains a value $(=0.11)$ slightly lower than the value in the high- $T$ PM state $(=0.13)$. Below $260 \mathrm{~K}$, $A_{10}$ shows another sharp fall, which can easily be assigned to the second magnetic transition occurring at $T_{C M}$. The most remarkable observation is the sluggish rise (as opposed to the sharp change at magnetic Curie points) of $A_{10}$ with decreasing $T$ below $T_{B}$. This is an indication of the loss of magnetic order, and it nicely fits with the conjecture of a glassy magnetic ground state of the system. It is evident that even at the lowest temperature, the value of $A_{10}(=0.03)$ is much smaller than the value observed in the high- $T$ PM state. It is worthwhile to mention that across the transition from PM to a magnetically ordered phase, one would expect the relaxing part of asymmetry to decrease by $\frac{1}{3}$ for a powder/polycrystalline sample in the ZF measurement. The residual $\frac{2}{3}$ component in the time domain data will correspond to the muon's damped oscillations due to the internal magnetic field $[27,28]$. In the present case, the drop in total initial asymmetry $\left(A_{0}=A_{10}+A_{20}\right)$ is significantly lower than $\frac{1}{3}$ just below $T_{C A}$. This may be due to the fast component of the longitudinal relaxation. We also do not observe any oscillation in the time domain data, which can be ascribed to the fact that the frequency of oscillation may be too high to observe in the spectrometer at ISIS due to the large internal field from $\mathrm{Mn}$.

The $T$ variations of $\lambda_{1}$ and $\lambda_{2}$ are plotted in Fig. 3(b), and they show well-defined peaks at $165 \mathrm{~K}$. In our magnetization data, there is no anomaly around $165 \mathrm{~K}$. However, it should be kept in mind that the spin freezing at $T_{B}(=120 \mathrm{~K})$ might have been reflected at a higher temperature due to the fast timescale of the $\mu$ SR measurements.

We have fitted the LF relaxation curves recorded under 45 $\mathrm{kOe}$, and the $T$ variation of the resulting parameter $A_{10}$ is depicted in Fig. 4(a). For the LF data, we recorded relaxation during both heating and cooling. The variation of $L F A_{10}(T)$ traces a nature similar to that of the ZF one. However, it provides a few pieces of additional information, which are not obvious in the ZF data. First, the sharp rise in $A_{10}$ due to MPT gets shifted to lower temperatures under LF. It is well known that the magnetic field favors austenite and it reduces $T_{M S}$, which gets well reflected in our $\mu \mathrm{SR}$ data. Second, clear thermal hysteresis is seen in $A_{10}(T)$ around the martensitic transition occurring at $T_{M S}$. The hysteresis is present in the plot of $\lambda_{1}$ and $\lambda_{2}$ as well [Fig. 4(b)], and it can be accounted for by the first-order nature of the structural transition. A second thermal hysteresis is present just below $T_{C M}$, which can be traced back to the similar thermal hysteresis observed in the bulk magnetization data (see Fig. 1). Such hysteresis may be linked to the first-order nature of the magnetic transition at $T_{C M}$.

Apart from the double-exponential fitting [Eq. (1)], we have used the stretched exponential function [as described by Eq. (2)] to fit the time domain data. A stretched exponential form of muon depolarization is generally expected both above and below the spin-freezing temperature $T_{g}$. For a simple PM to $\mathrm{SG}$ transition, $\beta$ attains a value close to unity at a temperature well above $T_{g}$. On cooling towards $T_{g}, \beta$ decreases [36]. It has been found that for so-called concentrated canonical spin glasses (in which there is a distribution of the frequency of fluctuation of the local magnetic field), $\beta$ attains a value of $\frac{1}{3}$ at $T_{g}$ [34].

We find that both the ZF and LF relaxation data can also be fitted well with a stretched exponential function as described in Eq. (2) below about $150 \mathrm{~K}$. In fact the quality of the fittings 


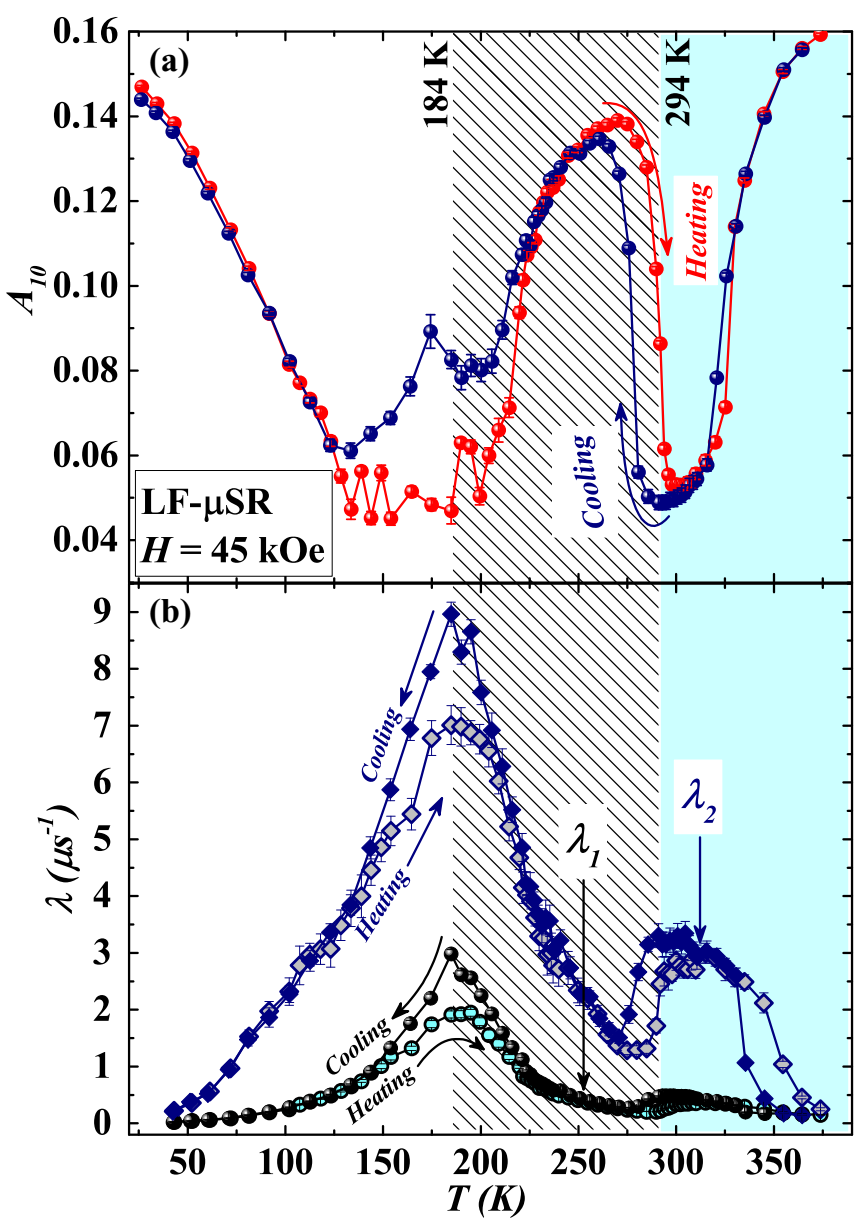

FIG. 4. (a) The muon asymmetry $A_{1}$ as a function of temperature measured in $45 \mathrm{kOe}$ of longitudinal magnetic field. (b) The thermal variation of the muon depolarization rate $\lambda_{1}$ and $\lambda_{2}$ at $H=45 \mathrm{kOe}$.

in the temperature range $30-150 \mathrm{~K}$ is found to be better (which is evident from the lower values of $\chi^{2}$ of the fits) in the case of the stretched exponential compared to two exponential functions. However, stretched exponential fitting becomes poorer above $150 \mathrm{~K}$, and it fails to converge with physically meaningful values of the fitting parameters. In Figs. 5(a), 5(b) and 5(c), we show the $T$ variations of parameters $A_{0}$, $\beta$, and $\lambda$, respectively, only below 150 , which were obtained by fitting the ZF and LF relaxation data recorded while the sample was being cooled. The initial asymmetry $A_{0}$ shows a rise below $150 \mathrm{~K}$, similar to the behavior of $A_{10}$ obtained from the two-exponential model. The exponent $\beta$ decreases as we approach $T_{B}$ from high temperature and shows a minimum at $120 \mathrm{~K}$ [Fig. 3(c)]. The values of $\beta$ at $T_{B}$ are found to be $\beta(\mathrm{ZF})_{T_{B}}=0.33(4)$ and $\beta(\mathrm{LF})_{T_{B}}=0.30(6)$. These values are fairly close to the $\beta=\frac{1}{3}$ law (particularly, the ZF one) for concentrated metallic SG. Below $T_{B}, \beta$ rises again and attains a value of 0.62 at $30 \mathrm{~K}$. On the other hand, $\lambda$ shows a decreasing trend on cooling along with a peaklike feature at $T_{B}$.

\section{B. Neutron powder diffraction measurements}

Figures 6(a), 6(b) and 6(c) show the high-resolution NPD data measured at different temperatures. The sample was first

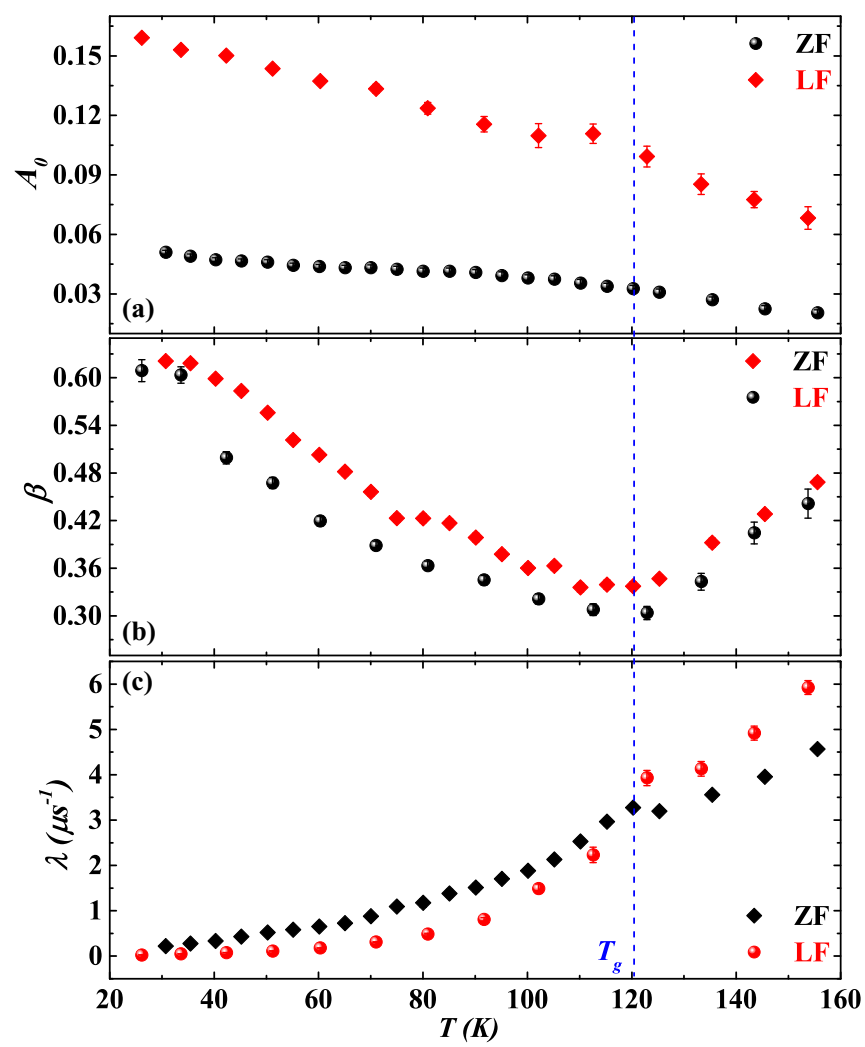

FIG. 5. The temperature variation of (a) initial asymmetry $A_{0}$, (b) the exponent $\beta$, and (c) relaxation rate $\lambda$ as obtained from fitting the time domain data using the stretched exponential function as described in Eq. (2). Both ZF and LF (50 kOe) data are represented in the plots.

heated to $363 \mathrm{~K}$ (which is well above $T_{M S}$ and $T_{C A}$ ), and diffraction data were recorded while cooling from 363 to $8 \mathrm{~K}$ within the closed-cycle refrigerator. The diffraction pattern at $363 \mathrm{~K}$ can be well indexed by the cubic $L 2_{1}$ structure with space group $F m \overline{3} m$, as expected for a pure austenitic phase [see Fig. 6(a)]. At $363 \mathrm{~K}$, the sample is in the PM state, and a good refinement is obtained by considering only the nuclear contribution of the cubic austenite with $L 2_{1}$ geometry. The refined cubic lattice parameter is found to be $a_{c}=5.991(1) \AA$. On cooling below $T_{M S}$, the cubic peaks start to disappear, along with the appearance of martensitic peaks [see the NPD data at $275 \mathrm{~K}$ in Fig. 5(c)].

At $8 \mathrm{~K}$, the data can be described by a single orthorhombic phase, as shown in Fig. 6(b). We do not observe any wellresolved magnetic superlattice reflection, which matches well with the previous report [7]. This rules out the possibility of an ordered AFM state below $T_{M S}$. In the case of related Ni-Co-Mn-Ga based Heusler alloys, a distinct AFM state was observed below the MPT [38]. A stable refinement is achieved assuming the nuclear phase comes from Pmma and one magnetic phase with propagation vector $k=(0,0,0)$. Here we have assumed that the ordered moments arise from the Mn atoms residing at only the $2 a$ and $2 f$ positions and neglected any contribution from Ni. We have fitted our data with several possible options of collinear magnetic structure with $k=(0,0,0)$, and the best fit is obtained when the moments are aligned along the orthorhombic $c$ axis. Mn atoms, situated 

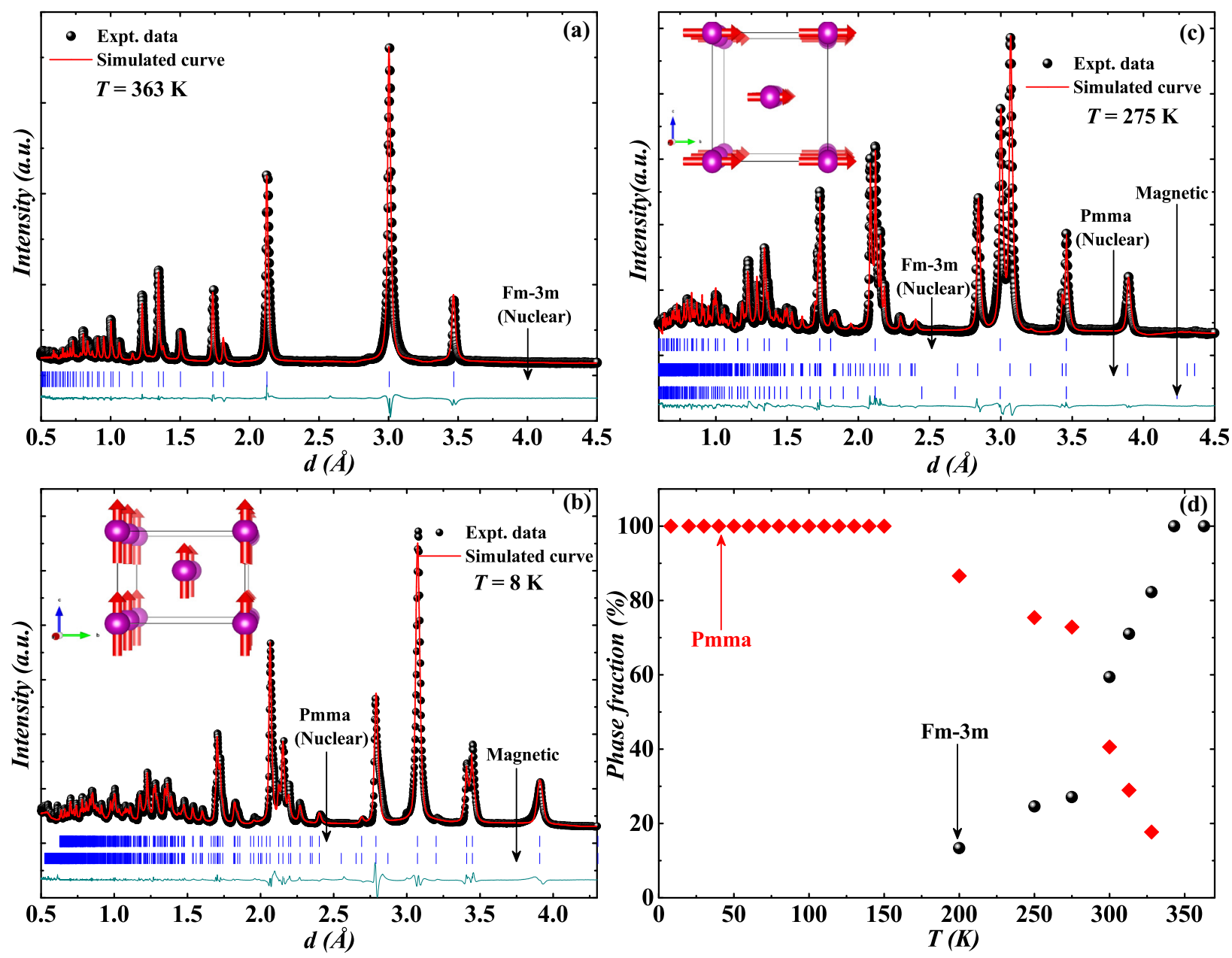

FIG. 6. The powder neutron diffraction data recorded at (a) 363, (b) 8, and (c) $275 \mathrm{~K}$. The solid lines are simulated by using the FULLPROF suite for Rietveld refinements. (d) The temperature variation of martensite and austenite phase fractions as obtained from the Rietveld refinement of the neutron diffraction data.

at $2 a$ and $2 f$ sites, have ordered moments $2.76 \mu_{B}$ and $2.30 \mu_{B}$, respectively (see Table I). Interestingly, unlike the previous report [7], we do not observe magnetic diffuse scattering in our data.

In Fig. 6(c), we plot NPD data for an intermediate temperature of $275 \mathrm{~K}$, where both $F m \overline{3} m$ (austenite) and Pmma (martensite) phases coexist, and we consider both the phases to refine the diffraction data. As evident from our $\mu$ SR data, the asymmetry rises below $T_{M S}$, indicating a PM martensitic phase. However, the residual austenite fraction may still be

TABLE I. Magnetic structure of the orthorhombic martensite (Pmma) as obtained from the powder neutron diffraction measurements at $8 \mathrm{~K}$. Magnetic moment is fixed along the [001] direction; $\chi^{2}$ of the fit $=5.45$.

\begin{tabular}{lccccc}
\hline \hline Atom & Site & $X$ & $Y$ & $Z$ & Moment (units of $\mu_{B}$ ) \\
\hline Mn & $2 a$ & 0 & 0 & 0 & $2.761(6)$ \\
& & 0.5 & 0 & 0 & $2.302(5)$ \\
Mn & $2 f$ & 0.25 & 0.5 & 0.574 & \\
& & 0.75 & 0.5 & 0.574 & \\
\hline \hline
\end{tabular}

present in the sample with an ordered FM state. Our effort to fit $275 \mathrm{~K}$ data considering only the nuclear contributions coming from cubic and orthorhombic phases does not provide a good convergence. A better fit is obtained when the FM contribution from the cubic phase is taken into consideration. Figure 6(c) shows the experimental data as well as the refinements. The ratio of the volume fraction of the cubic and orthorhombic phases is found to be $\frac{2}{3}: \frac{1}{3}$. This indicates that below $T_{M S}$, the major phase fraction is martensite, although a sizable amount of austenite is still present. The cubic and orthorhombic lattice parameters are found to be $a_{c}=$ 5.991(7) $\AA$ and $a_{o}=8.613(4) \AA, b_{o}=5.675(6) \AA$, and $c_{o}=$ 4.360(5) $\AA$, respectively. The ordered Mn moments are found to be $1.78 \mu_{B}$ and $1.13 \mu_{B}$ at the $4 a$ and $4 b$ sites, respectively (see Table II). It should be noted that the moment value at the $4 b$ site is lower than that obtained in a previous study on a similar alloy [7]. This may be due to the fact that our sample has a slightly different composition.

Interestingly, a fraction of high- $T$ austenite continues to exist over a wide temperature range well below $T_{M S}$. Eventually, the reflections due to the austenite disappear when the sample is cooled below $200 \mathrm{~K}$. In order to determine the $T$ variation of phase fraction, we performed structural 
TABLE II. Magnetic structure data for the cubic austenite $(F m \overline{3} m)$ at $275 \mathrm{~K}$ as obtained from the powder neutron diffraction measurements. Magnetic moment is fixed along the [010] direction; $\chi^{2}$ of the fit $=6.15$.

\begin{tabular}{lccccc}
\hline \hline Atom & Site & $X$ & $Y$ & $Z$ & Moment (units of $\mu_{B}$ ) \\
\hline Mn & $4 a$ & 0 & 0 & 0 & $1.780(7)$ \\
Mn & $4 b$ & 0.5 & 0.5 & 0.5 & $1.131(5)$ \\
\hline \hline
\end{tabular}

refinements of the NPD data at different temperatures between 8 and $363 \mathrm{~K}$. Considering the coexistence of the cubic and the orthorhombic phases, the data were refined using two phases. Figure 6(d) shows how the fraction of orthorhombic and cubic phases changes with $T$. As expected, the cubic fraction diminishes rapidly on cooling and disappears below $200 \mathrm{~K}$. The orthorhombic phase fraction, on the other hand, increases monotonically and almost saturates below $150 \mathrm{~K}$. It should be noted that the thermal hysteresis in the magnetization and $\mu$ SR data disappears below $150 \mathrm{~K}$. Therefore, $150 \mathrm{~K}$ can be assigned as the culminating point of MPT, below which the system attains a stable martensite fraction. The crystallographic parameters of the martensite at $8 \mathrm{~K}$, as obtained from our NPD data, are depicted in Table III.

\section{DISCUSSION}

The complex magnetic phases of the studied alloy get reflected in the $\mu$ SR data, and in association with the NPD result, it significantly clarifies the prevailing doubts on the magnetic states of such Ni-Mn- $Z$ based MSMAs. We observe that the values of $A_{10}$ obtained from the $\mu \mathrm{SR}$ data (both $\mathrm{ZF}$ and LF) show a sharp rise on cooling below $T_{M S}$, which continues until the second magnetic transition at $T_{C M}$ is attained. The bulk magnetic measurements (as depicted in Fig. 1) indicate a rapid fall of $M$ below $T_{M S}$, which could be due to the development of (i) a long-range ordered AFM state, (ii) a long-range ordered FM state, albeit with a highly reduced Mn moment, (iii) a state with short-range AFM correlations, (iv) ordered FM clusters in the backdrop of a PM state, or (v) a pure PM state.

This sharp rise in $A_{10}$ below $T_{M S}$ summarily rejects cases (i) and (ii), as long-range order should not be accompanied by increasing asymmetry. Therefore, we are left with options (iii), (iv), and (v). If we look at the variation of $A_{10}(T)$, the asymmetry does not fully attain its austenite PM state value just below $T_{M S}$. Therefore, the scenario of a pure PM state can

TABLE III. Crystallographic parameters of the sample at $8 \mathrm{~K}$ (Pmma) as obtained from the refinements.

\begin{tabular}{lccllc}
\hline \hline Atom & Site & $X$ & \multicolumn{1}{c}{$Y$} & \multicolumn{1}{c}{$Z$} & \multicolumn{1}{c}{$B_{\text {iso }}$} \\
\hline $\mathrm{Ni}$ & $4 h$ & 0.0000 & $0.249(6)$ & 0.5000 & $1.229(7)$ \\
$\mathrm{Ni}$ & $4 k$ & 0.2500 & $0.2484(4)$ & $0.091(5)$ & $0.544(4)$ \\
$\mathrm{Mn}$ & $2 a$ & 0.0000 & 0.0000 & 0.0000 & $1.616(6)$ \\
$\mathrm{Mn}$ & $2 f$ & 0.2500 & 0.5000 & $0.574(7)$ & $1.500(5)$ \\
$\mathrm{Mn}$ & $2 b$ & 0.0000 & 0.5000 & 0.0000 & $0.990(4)$ \\
$\mathrm{Mn}$ & $2 e$ & 0.2500 & 0.0000 & $0.562(6)$ & $0.990(4)$ \\
$\mathrm{Sn}$ & $2 b$ & 0.0000 & 0.5000 & 0.0000 & $0.990(4)$ \\
$\mathrm{Sn}$ & $2 e$ & 0.2500 & 0.0000 & $0.562(6)$ & $0.990(4)$ \\
\hline \hline
\end{tabular}

be excluded. The magnetic state just below $T_{M S}$ can be either due to the presence of the AFM phase fraction or associated with the residual FM austenite which remained untransformed even below $T_{M S}$. In our NPD data, we find a clear signature of this cubic austenite down to $200 \mathrm{~K}$. Therefore, the mismatch of $A_{10}$ at $264 \mathrm{~K}\left(\approx T_{C M}\right)$ and $350 \mathrm{~K}$ in the ZF $\mu$ SR data is likely to be associated with this cubic FM fraction. The variation of $A_{10}(T)$ in the LF $\mu$ SR data is somewhat similar, although the signature of $T_{M S}$ has shifted slightly to lower temperature. This is due to the fact that an external magnetic field prefers the ferromagnetically ordered austenite $[39,40]$. Logically, the most probable scenario is case (iv), where the transformed martensite is PM (the major phase), residing along with the untransformed FM austenite (the minor phase).

The thermal hysteresis around the martensitic transition is expected, and it is present in the $\mu \mathrm{SR}$ data too. Interestingly, both $M(T)$ and $A_{10}(T)$ show another thermal hysteresis between 150 and $225 \mathrm{~K}$. There are several reports on twostep martensitic transition, where a second intermartensite transformation occurs [41,42]. In the case of one such NiMn-In based alloy, the intermartensite transition was found to occur just below $T_{C M}$, and it was assigned to a transformation from the $10 \mathrm{M}$ to $14 \mathrm{M}$ structure [43]. Here 10M (14M) stands for modulated martensite, which originates from the periodic variation of atomic layers so that the original position is regained after every 10 (14) repetitions of atomic planes. We carefully looked at the NPD data in this temperature range; however, no anomaly was detected in the form of peak splitting or the appearance of additional reflections. The magnetic transition at around $T_{C M}$ is certainly a first-order one; however, it may be an isostructural one where the lattice symmetry remains unaltered.

The most important observation in the present work is the signature of $T_{B}$ in the $\mu \mathrm{SR}$ data. As is evident [see Figs. 3(a) and 4(a)], the initial asymmetry shows a rise below $T_{B}$, indicating the loss of magnetic order in the system. Notably, this rise is present irrespective of the fitting function used (two-exponential or stretched exponential). If we look at the variation of $\beta$ [see Fig. 5(b)], it shows a minimum at $T_{B}=$ $120 \mathrm{~K}$ with the value of $\beta$ close to 0.33 . In addition, $\lambda$ shows a weak peak [see Fig. 5(b)] around $T_{B}$. Considering the glassy magnetic state observed in the family of Ni-Mn-Sn alloys below $T_{B}$, we can assign $T_{B}$ to be the spin-freezing temperature of the presently studied sample.

It is now pertinent to discuss the nature and origin of the SG ground state. From our NPD data, we observe a single-phase orthorhombic martensite at the base temperature. Therefore, the spin freezing is not related to the presence of the minority cubic phase in the system. In Ni-Mn- $Z$ based MSMAs, the sign and strength of magnetic interaction depend strongly on the Mn-Mn bond distance. Below $T_{M S}$, the intersite Mn$\mathrm{Mn}^{\prime}$ distance decreases, paving the way for enhanced AFM correlation $[8,11,14,44]$. In addition, chemical and lattice disorders play an important role in determining the magnetic ground state of these materials [11]. The AFM correlations between $\mathrm{Mn}$ and $\mathrm{Mn}^{\prime}$ (particularly below $T_{M S}$ ), the Mn-Mn FM correlations, and the presence of disorder eventually lead to spin freezing below $T_{B}$. The $\mathrm{Mn}^{\prime}$ atoms are substituted randomly in the Sn site, which can give rise to the random occurrence of FM and AFM bonds. From our analysis of 
the NPD data, it is evident that the AFM correlation is short range in nature; that is, it does not give rise to a long-range ordered AFM state. Nevertheless, the ground state does show long-range FM order.

In general for a PM to SG transition, the value of $\beta$ in the stretched exponential fitting assumes a constant value below the spin-freezing temperature due to the residual fluctuation of the frozen state [35]. In contrast to the usual observation, the value of $\beta$ increases below $T_{B}$ and attains a value of 0.62 at $30 \mathrm{~K}$. The main reason for such anomalous behavior of $\beta$ lies in the reentrant character of the SG state, where spin freezing takes place on top of a long-range FM state. Interestingly, very similar $T$ dependence of $\beta$ was reported in the case of $\mathrm{Pb}\left(\mathrm{Fe}_{1 / 2} \mathrm{Nb}_{1 / 2}\right) \mathrm{O}_{3}$, where $\beta$ shows a minimum at $T_{g}=20 \mathrm{~K}$ and approaches unity on further cooling [37]. It has been argued that with the critical slowing down of the SG fluctuations at $T_{g}$, muons start to sense the noncritical fluctuations of the long-range ordered state below $T_{g}$, leading to an increase in $\beta$. By analogy, the rise in $\beta$ below $T_{B}$ in the studied Ni-Mn-Sn alloy can be accounted for by the presence of a long-range ordered FM phase with the SG state side by side. This is corroborated by the NPD data, where FM order is indeed present at $8 \mathrm{~K}$. The weak nature of the peak in the $\lambda(T)$ data (an ideal spin glass should show a sharp peak at the spin-freezing temperature) may be due to the presence of this FM ordered state alongside the SG phase. We may rule out the possibility of a cluster-glass-like ground state in this composition, as both NPD and $\mu$ SR give strong evidence for an ordered FM sate.

In conclusion, the present work successfully discerns a few ambiguities related to the magnetic phase diagram of the Ni-Mn-Sn alloy system. Based on our study on a particular $\mathrm{Ni}-\mathrm{Mn}-\mathrm{Sn}$ alloy, it is evident that the alloy assumes a PM state just below the martensitic transition. The $\mu \mathrm{SR}$ result identifies two long-range magnetic ordering temperatures, $T_{C A}$ and $T_{C M}$, and they are found to be ferromagnetic in nature. This work categorically justifies the view that the magnetic anomaly at $T_{C M}$ in the martensitic state indeed corresponds to the onset of a long-range ordered state. Most importantly, this work identifies that the system transforms into a partially disordered magnetic phase below the exchange bias blocking temperature, which can be characterized by the coexistence of ordered FM and frozen-spin-glass states. The remarkable phenomenon of exchange bias observed in Ni-Mn- $Z$ alloys is due to the coupling between the interfacial spins of SG and FM phases.

\section{ACKNOWLEDGMENTS}

The work is supported by the India-RAL collaborative project (SR/NM/Z-07/2015). J.S. wishes to acknowledge EU's Horizon 2020 research and innovation program under the Marie Skłodowska-Curie Grant Agreement (No. 665593) awarded to the STFC, United Kingdom.
[1] R. Kainuma, Y. Imano, W. Ito, Y. Sutou, H. Morito, S. Okaoto, O. Kitakami, K. Oikawa, A. Fujita, T. Kanomata, and K. Ishida, Nature (London) 439, 957 (2006).

[2] T. Krenke, E. Duman, M. Acet, E. F. Wassermann, X. Moya, L. Mañosa, and A. Planes, Nat. Mater. 4, 450 (2005).

[3] R. Kainuma, K. Oikawa, W. Ito, Y. Sutou, T. Kanomata, and K. Ishida, J. Mater. Chem. 18, 1837 (2008).

[4] L. Mañosa, X. Moya, A. Planes, T. Krenke, M. Acet, and E. F. Wassermann, Mater. Sci. Eng. A 481-482, 49 (2008).

[5] S. Chatterjee, S. Giri, and S. Majumdar, and S. K. De, Phys. Rev. B 77, 012404 (2008).

[6] L. Mañosa, D. González-Alonso, A. Planes, E. Bonnot, M. Barrio, J.-L. Tamarit, S. Aksoy, and M. Acet, Nat. Mater. 9, 478 (2010).

[7] P. J. Brown, A. P. Gandy, K. Ishida, R. Kainuma, T. Kanomata, K. U. Neumann, K. Oikawa, B. Ouladdiaf, and K. R. A. Ziebeck, J. Phys.: Condens. Matter 18, 2249 (2006).

[8] V. D. Buchelnikov, P. Entel, S. V. Taskaev, V. V. Sokolovskiy, A. Hucht, M. Ogura, H. Akai, M. E. Gruner, and S. K. Nayak, Phys. Rev. B 78, 184427 (2008).

[9] M. Ye, A. Kimura, Y. Miura, M. Shirai, Y. T. Cui, K. Shimada, H. Namatame, M. Taniguchi, S. Ueda, K. Kobayashi, R. Kainuma, T. Shishido, K. Fukushima, and T. Kanomata, Phys. Rev. Lett. 104, 176401 (2010).

[10] C. L. Tan, Y. W. Huang, X. H. Tian, J. X. Jiang, and W. Cai, Appl. Phys. Lett. 100, 132402 (2012).

[11] V. V. Sokolovskiy, V. D. Buchelnikov, M. A. Zagrebin, P. Entel, S. Sahoo, and M. Ogura, Phys. Rev. B 86, 134418 (2012).
[12] H. B. Xiao, C. P. Yang, R. L. Wang, V. V. Marchenkov, and X. Luo, J. Appl. Phys. 115, 203703 (2014).

[13] C.-M. Li, Q.-M. Hu, R. Yang, B. Johansson, and L. Vitos, Phys. Rev. B 92, 024105 (2015).

[14] K. R. Priolkar, P. A. Bhobe, D. N. Lobo, S. W. D’Souza, S. R. Barman, A. Chakrabarti, and S. Emura, Phys. Rev. B 87, 144412 (2013).

[15] R. Y. Umetsu, R. Kainuma, Y. Amako, Y. Taniguchi, T. Kanomata, K. Fukushima, A. Fujita, K. Oikawa, and K. Ishida, Appl. Phys. Lett. 93, 042509 (2008).

[16] P. J. Brown, A. P. Gandy, R. Kainuma, T. Kanomata, K. U. Neumann, K. Oikawa, B. Ouladdiaf, A. Sheikh, and K. R. A. Ziebeck, J. Phys.: Condens. Matter 23, 456004 (2011).

[17] S. Aksoy, M. Acet, P. P. Deen, L. Mañosa, and A. Planes, Phys. Rev. B 79, 212401 (2009).

[18] S. Chatterjee, S. Giri, S. K. De, and S. Majumdar, Phys. Rev. B 79, 092410 (2009).

[19] D. Y. Cong, S. Roth, J. Liu, Q. Luo, M. Pötschke, C. Hürrich, and L. Schultz, Appl. Phys. Lett. 96, 112504 (2010).

[20] B. M. Wang, Y. Liu, P. Ren, B. Xia, K. B. Ruan, J. B. Yi, J. Ding, X. G. Li, and L. Wang, Phys. Rev. Lett. 106, 077203 (2011).

[21] R. Y. Umetsu, A. Fujita, W. Ito, T. Kanomata, and R. Kainuma, J. Phys.: Condens. Matter 23, 326001 (2011).

[22] M. K. Ray, K. Bagani, P. K. Mukhopadhyay, and S. Banerjee, Europhys. Lett. 109, 47006 (2015).

[23] S. Pramanick, S. Chatterjee, S. Giri, and S. Majumdar, Appl. Phys. Lett. 105, 112407 (2014).

[24] S. Pramanick, S. Chattopadhyay, S. Giri, S. Majumdar, and S. Chatterjee, J. Appl. Phys. 116, 083910 (2014). 
[25] FULLPROF, https://www.ill.eu/sites/fullprof/.

[26] S. J. Blundell, Contemp. Phys. 40, 175 (1999).

[27] D. H. Ryan, J. van Lierop, and J. M. Cadogan, J. Phys.: Condens. Matter 16, S4619 (2004).

[28] P. Dalmas de Réotier and A. Yaouanc, J. Phys.: Condens. Matter 9, 9113 (1997).

[29] R. H. Heffner, J. E. Sonier, D. E. MacLaughlin, G. J. Nieuwenhuys, G. Ehlers, F. Mezei, S.-W. Cheong, J. S. Gardner, and H. Röder, Phys. Rev. Lett. 85, 3285 (2000).

[30] Y. Kawasaki, T. Minami, M. Izumi, Y. Kishimoto, T. Ohno, K. H. Satoh, A. Koda, R. Kadono, J. L. Gavilano, H. Luetkens, T. Nakajima, and Y. Ueda, Phys. Rev. B 86, 125141 (2012).

[31] R. H. Heffner, J. E. Sonier, D. E. MacLaughlin, G. J. Nieuwenhuys, G. M. Luke, Y. J. Uemura, W. Ratcliff, II, S.-W. Cheong, and G. Balakrishnan, Phys. Rev. B 63, 094408 (2001).

[32] H. Guo, K. Manna, H. Luetkens, M. Hoelzel, and A. C. Komarek, Phys. Rev. B 94, 205128 (2016).

[33] M. Hiroi, T. Hisamatsu, T. Suzuki, K. Ohishi, Y. Ishii, and I. Watanabe, Phys. Rev. B 88, 024409 (2013).

[34] I. A. Campbell, A. Amato, F. N. Gygax, D. Herlach, A. Schenck, R. Cywinski, and S. H. Kilcoyne, Phys. Rev. Lett. 72, 1291 (1994).
[35] Y. Fudamoto, K. M. Kojima, M. I. Larkin, G. M. Luke, J. Merrin, B. Nachumi, Y. J. Uemura, M. Isobe, and Y. Ueda, Phys. Rev. Lett. 83, 3301 (1999).

[36] J. Teyssier, E. Giannini, V. Guritanu, R. Viennois, D. van der Marel, A. Amato, and S. N. Gvasaliya, Phys. Rev. B 82, 064417 (2010).

[37] G. M. Rotaru, B. Roessli, A. Amato, S. N. Gvasaliya, C. Mudry, S. G. Lushnikov, and T. A. Shaplygina, Phys. Rev. B 79, 184430 (2009).

[38] F. Orlandi, S. Fabbrici, F. Albertini, P. Manuel, D. D. Khalyavin, and L. Righi, Phys. Rev. B 94, 140409(R) (2016).

[39] K. Koyama and K. Watanabe, Appl. Phys. Lett. 88, 132505 (2006).

[40] A. Planes, L. Mañosa, and M. Acet, J. Phys.: Condens. Matter 21, 233201 (2009).

[41] G. Fan, W. Chen, S. Yang, J. Zhu, X. Ren, and K. Otsuka, Acta Mater. 52, 4351 (2004).

[42] M. C. Carroll, Ch. Somsen, and G. Eggeler, Scr. Mater. 50, 187 (2004).

[43] Y. J. Huang, Q. D. Hu, J. Liu, L. Zeng, D. F. Zhang, and J. G. Li, Acta Mater. 61, 5702 (2013).

[44] P. Borgohain and M. B. Sahariah, J. Phys.: Condens. Matter 27, 175502 (2015). 